\title{
Privatisierung des Krieges und Auslagerung öffentlicher Dienstleistungen : Herausforderungen für die humanitäre Aktion
}

Gilles Carbonnier

\section{OpenEdition}

Electronic version

URL: http://journals.openedition.org/sjep/479

DOI: $10.4000 /$ sjep.479

ISSN: 1663-9677

Publisher

Institut de hautes études internationales et du développement

Printed version

Date of publication: 1 octobre 2005

Number of pages: 121-129

ISBN: 2-88247-059-2

ISSN: $1660-5926$

\section{Electronic reference}

Gilles Carbonnier, «Privatisierung des Krieges und Auslagerung öffentlicher Dienstleistungen

Herausforderungen für die humanitäre Aktion », Schweizerisches Jahrbuch für Entwicklungspolitik [Online], 24-2 | 2005, Online erschienen am: 08 Juni 2010, abgerufen am 08 September 2020. URL: http://journals.openedition.org/sjep/479; DOI : https://doi.org/10.4000/sjep.479 


\title{
Privatisierung des Krieges und Auslagerung öffentlicher Dienstleistungen: Herausforderungen für die humanitäre Aktion
}

\author{
Gilles Carbonnier*
}

\section{Einführung}

Der Irakkrieg hat ein Schlaglicht auf die immer wichtigere Rolle privater Militärfirmen (,private military companies“) in bewaffneten Konflikten geworfen. Die Beispiele amerikanischer Staatsbürger, die für die Firma Blackwater arbeiteten und an Gefechten in Falludscha teilnahmen, sowie von Beschäftigten der CACI International und der Titan Corp., welche an den Misshandlungen der Häftlinge im Gefängnis von Abu Ghraib beteiligt waren, verdeutlichen die immer umfassenderen Aufgaben, die solchen Firmen anvertraut werden: Ausbildung und Training der Sicherheitskräfte, logistische Unterstützung, Verhöre und Nachrichtendienste, Absicherung strategischer Orte, Personennahschutz, direkte Beteiligung an den Feindseligkeiten, usw. Eine solche Privatisierung des harten Kerns der Staatsaufgaben wirft grundlegende rechtliche und humanitäre Fragen auf.

Gleichzeitig vergeben die Staaten immer mehr öffentliche Dienste, zum Beispiel die Versorgung der Bevölkerung mit Trinkwasser, an private Auftragnehmer. Dieses Phänomen der Untervertragsvergabe und der Privatisierung ist vor dem Hintergrund des Legitimitätsdefizits der öffentlichen Einrichtungen und der Strukturschwächen in zahlreichen Entwicklungsländern zu sehen, die es den Staaten nicht mehr erlauben, ihre Vorrechte voll und ganz auszuüben und im gesamten Hoheitsgebiet vorzugehen. In bestimmten Regionen Nigerias oder Indonesiens beispielsweise sind die Erdöl- und Bergbaugesellschaften in Bereichen wie Erziehung, Gesundheit, Sicherheit oder Infrastrukturen zu den grössten Geldgebern geworden.

Mehrere Regierungen, internationale Institutionen und Nichtregierungsorganisationen (NGO) erwarten von Privatunternehmen, dass sie bei der Bewältigung globaler Probleme wie Armut, Wassermangel, Klimaerwärmung oder bewaffneten Konflikten mehr Verantwortung übernehmen. Das beginnende 21. Jahrhundert steht somit im Zeichen einer tief greifenden Infragestellung der Vorrechte und Verantwortlichkeiten der öffentlichen Institutionen gegenüber dem Privatsektor.

* Wirtschaftlicher Berater und Koordinator für die Beziehungen mit dem Privatsektor, Internationales Komitee vom Roten Kreuz (IKRK). Der Autor dankt Emanuela-Chiara Gillard für die juristischen Ratschläge zur Frage der privaten Militärfirmen.

Bei diesem Kapitel handelt es sich um einen Auszug aus einem Artikel, der im Dezember 2004 in der Revue internationale de la Croix-Rouge Nr. 856 unter folgendem Titel erschien: „Privatisations, soustraitance et partenariats public-privé: Charity.com ou Business.org?"“. Der vorliegende Artikel gibt die Meinungen des Autors wieder, die nicht unbedingt mit der Position des Internationalen Komitees vom Roten Kreuz übereinstimmen. 
Diese Entwicklungen rufen die humanitären Organisationen auf den Plan: Die Krisensituationen verschärfen tendenziell die Probleme, welche die Privatisierung und die Auslagerung von angestammten Aufgaben des Staates, mit bisweilen dramatischen Folgen für die betroffenen Bevölkerungen, verursachen können.

Im vorliegenden Artikel werden zuerst die rechtlichen und humanitären Fragen betreffend das Aufkommen der privaten Militärfirmen untersucht. Anschliessend befassen wir uns mit der Privatisierung und der Auslagerung von Dienstleistungen im Zusammenhang mit der Wasserversorgung, der Verwaltung von Haftanstalten und der Gesundheitspflege.

\section{Privatisierung militärischer Aufgaben}

Private Sicherheitskräfte gab es bereits in der Antike, sie spielten im Mittelalter eine wichtige Rolle und sind seit dem Ende des Kalten Krieges wieder in vollem Aufschwung. Experten beziffern den Umsatz der privaten Militärfirmen im Jahr 2002 auf bereits über 100 Milliarden Dollar.

Privatunternehmen haben zum Hauptziel, die grösstmögliche Rentabilität für das investierte Kapital zu garantieren. Dies gilt auch für die privaten Militärfirmen. Dieses Ziel der Gewinnmaximierung wirft im Rahmen von bewaffneten Konflikten ethische Fragen auf. So können zum Beispiel Kosteneinsparungen dazu führen, dass eine Firma die Schulung ihres Personals in Bezug auf die Verhältnismässigkeit und die Zurückhaltung bei Gewaltanwendung vernachlässigt. Einer rein ökonomischen Argumentation folgend, weisen bestimmte Beobachter darauf hin, dass diese Gesellschaften ein Interesse daran haben könnten, dass die Nachfrage nach ihren Dienstleistungen - mit der wachsenden Verunsicherung oder sogar mit der Zunahme der bewaffneten Konflikte - steigt.

Für Peter Singer ${ }^{1}$, Experte der Brookings Institution in Washington, lässt sich der vermehrte Einsatz von privaten Militärfirmen nicht allein mit ökonomischem Rationalitätsdenken erklären, sondern hängt eher mit politischen Erfordernissen zusammen. Gemäss dem Autor muss in den USA das Pentagon den Kongress über seine Aufträge mit privaten Sicherheitsanbietern für Beträge unter 50 Millionen Dollar gar nicht benachrichtigen. Somit droht die Legislative ihre Kontrolle über Militäroperationen im Ausland teilweise zu verlieren, da die Exekutive direkt mit den privaten Sicherheitsfirmen Verträge über die Weitervergabe von Aufträgen abschliessen kann.

Das Aufkommen der privaten Militärfirmen und ihre Beziehungen zu den Staaten werfen eine Reihe juristischer Fragen auf. Die erste betrifft die Rechtsstellung dieser Gesellschaften und ihrer Mitarbeiter in bewaffneten Konflikten. Artikel 47 des ersten Zusatzprotokolls zu den Genfer Konventionen vom 12. August 1949 über den Schutz der Opfer internationaler bewaffneter Konflikte (Protokoll I) sieht vor, dass Söldner kein Recht auf den Kombattanten- oder Kriegsgefangenenstatus haben ${ }^{2}$, und präzisiert, dass der Begriff «Söldner» alle

1 Peter Singer, Corporate Warriors: The Rise of the Private Military Industry. The Privatization of War, Ithaca, Cornell University Press, 2003.

2 Dieser Artikel verbietet das Söldnerwesen nicht als solches, im Gegensatz zur Konvention der Organisation für Afrikanische Einheit vom 3. Juli 1977 über die Beseitigung des Söldnerwesens in Afrika 
Personen erfasst, welche sechs kumulative Kriterien erfüllen. Bei einer derart einschränkenden Definition geraten die Verantwortlichen von privaten Militärfirmen in der Praxis wohl nur mit grosser Unvorsichtigkeit und sehr viel Pech in die Lage, dass ihre Beschäftigten unter diese Kategorien fallen! Da solche Szenarien in der Praxis relativ selten vorkommen, wird Angestellten der privaten Militärfirmen, die an einem internationalen bewaffneten Konflikt beteiligt sind, entweder der Status von Zivilpersonen oder der eines Kombattanten zuerkannt. Im humanitären Völkerrecht gelten sie grundsätzlich als Zivilpersonen, sofern sie nicht de facto in die Streitkräfte einer Konfliktpartei integriert sind (vgl. Artikel 4 der Genfer Konvention vom 12. August 1949 über die Behandlung der Kriegsgefangenen; vgl. Artikel 43 des Protokolls I). Bei direkter Mitwirkung an den Feindseligkeiten verlieren sie indessen den Schutz, der Zivilpersonen vor Angriffen gewährt wird, und zwar für die Dauer ihrer Beteiligung (vgl. Artikel 51 des Protokolls I); sie behalten aber ihren Status als Zivilpersonen.

Das humanitäre Völkerrecht definiert die direkte Mitwirkung an Feindseligkeiten zwar nicht eindeutig, jedoch gehören bestimmte Aktivitäten der privaten Militärfirmen klar zu dieser Kategorie. Beispiele dafür sind der Einsatz von Waffengewalt zur Verteidigung eines Militärziels, die Ausrichtung und das Abschiessen von Lenkwaffen oder der Munitionstransport. Im zuletzt genannten Fall wäre vorstellbar, dass Lastwagenfahrer aus einkommensschwachen Ländern sich für gutes Geld der Privatfirmen bereit erklären, im Kontext eines bewaffneten Konflikts Militärmaterial zu transportieren, aber dabei nicht unbedingt wissen, dass sie nun gemäss dem humanitären Völkerrecht ein «legitimes» Angriffsziel bilden. Gleiches gilt für private Auftragnehmer, welche die Sicherheit von militärischen Objekten gewährleisten und bei einem Angriff auf diese Ziele Opfer von „Kollateralschäden“ werden.

Die Beschäftigten von privaten Militärfirmen gelten als Zivilpersonen, sofern und solange sie nicht in die Streitkräfte einer Konfliktpartei integriert sind. Mit der Häufung der privaten Sicherheitsanbieter vor Ort könnte es dabei immer schwieriger werden, in der Konfliktführung effektiv zwischen Zivilpersonen und Kombattanten zu unterscheiden. In der Praxis hätte dies eine Aufweichung des Schutzes der Zivilpersonen zur Folge.

Das Aufkommen dieser neuen Akteure stellt das Internationale Komitee vom Roten Kreuz (IKRK) vor ein zusätzliches Problem. Es muss gewährleisten, dass die Privatisierung von Aktivitäten, die herkömmlicherweise (zumindest in den letzten beiden Jahrhunderten) von regulären Armeen oder gegnerischen Kräften durchgeführt wurden, die von den bewaffneten Konflikten betroffenen Zivilbevölkerungen nicht beeinträchtigt. Deshalb ist es wesentlich, dass die Staaten ihre Verantwortung wahrnehmen und dafür sorgen, dass das humanitäre Völkerrecht respektiert wird - auch von privaten Sicherheitskräften, die in ihrem Hoheitsgebiet agieren, oder die sie für Auslandsoperationen einstellen. Der gemeinsame Artikel 1 der Genfer Konventionen von 1949 sieht dazu vor, dass die hohen Vertragsparteien sich verpflichten, diese Konventionen unter allen Umständen einzuhalten und ihre Einhaltung durchzusetzen.

sowie zur Internationalen Konvention vom 4. Dezember 1989 über Anwerbung, Einsatz, Finanzierung und Ausbildung der Söldner. 
Die Völkerrechtskommission der Vereinten Nationen verfasste einen Entwurf zu Artikeln über die Verantwortung des Staates für international unrechtmässige Handlungen, der von der Generalversammlung im Jahr 2001 angenommen wurde. Der Entwurf bekräftigt einen Grundsatz des internationalen öffentlichen Rechts: Die Staaten haften nicht nur für Handlungen ihrer eigenen Organe, sondern auch für die Handlungen aller Personen oder Dienststellen, die zwar kein staatliches Organ bilden, aber vom Recht des betroffenen Staates ermächtigt werden, öffentliche Aufgaben und Machtbefugnisse auszuüben (Art. 5). Artikel 8 hält zudem fest, dass das Verhalten einer Person oder Personengruppe gemäss dem Völkerrecht als Handlung des Staates gilt, sofern die Person oder Personengruppe dabei tatsächlich auf Anordnung oder Weisung bzw. unter der Kontrolle dieses Staates handelt.

Somit können sich die Staaten nicht ihren Verpflichtungen im Rahmen des humanitären Völkerrechts entziehen, indem sie bestimmte Militäraktivitäten an private Gesellschaften vergeben. Wenn zum Beispiel ein Staat Kriegsgefangene in Haft hält und die Verwaltung der Haftanstalt einem privaten Unternehmen anvertraut, so muss er trotzdem gewährleisten, dass die Verwaltung der Gefängnisse den Normen der III. Genfer Konvention entspricht. Der Staat kann für gravierende Verstösse nicht allein die privaten Gesellschaften verantwortlich machen. Auch haften Staaten, die private Militärfirmen engagieren, für Verletzungen des humanitären Völkerrechts durch diese Gesellschaften und ihre Mitarbeiter.

Die privaten Militärfirmen und ihr Personal sind für ihre etwaigen Verstösse gegen das humanitäre Völkerrecht ebenfalls verantwortlich. Diese Haftung steht zwar eindeutig fest, jedoch lassen die Umsetzungsmechanismen, die es erlauben, ein Unternehmen vor Gericht zu bringen oder gegen die Angestellten, welche Verbrechen begangen haben, vorzugehen, zu wünschen übrig. Das Prinzip der zivilrechtlichen Haftung von Privatunternehmen ist in der Regel im Recht und in der Praxis fest verankert. Hingegen ist die strafrechtliche Haftung von Privatunternehmen viel beschränkter. Ebenso wird die strafrechtliche Haftung der Täter bei Verletzungen des humanitären Völkerrechts zwar klar anerkannt, jedoch kann es sich in der Praxis als schwierig erweisen, gegen die Täter gerichtlich vorzugehen. Die Gesellschaften und ihre Angestellten geniessen bisweilen in ihren Einsatzländern völlige Straffreiheit, zum Beispiel wenn das Justizsystem wegen des bewaffneten Konflikts lahmgelegt wird. Problematisch kann auch die Verfolgung der Schuldigen bei Verstössen gegen das humanitäre Völkerrecht in dem Land sein, in dem sich der Firmensitz der privaten Militärgesellschaft befindet, weil die strafbaren Handlungen im Ausland begangen wurden und die betroffenen Gerichte mitunter keine gebietsfremde Gerichtsbarkeit ausüben können, bzw. dies aus praktischen Gründen (z.B. fehlendes Beweismaterial) oder aus politischen Motiven nicht wünschen. Deshalb ist es dringend erforderlich, diese Fragen zu klären, um zu gewährleisten, dass Verletzungen des humanitären Völkerrechts - unabhängig von der Staatsbürgerschaft des Täters, von der Staatsbürgerschaft der Gesellschaft, für die er arbeitet, und vom Land, in welchem der Verstoss begangen wurde - geahndet werden. Die aktuelle juristische Grauzone darf nicht dazu führen, dass Mitarbeiter von privaten Militärfirmen, die gegen das humanitäre Völkerrecht verstossen, de facto straffrei ausgehen. 
Noch komplexer wird die Frage der Rechte und Verantwortlichkeiten aller von den Aktivitäten einer privaten Militärgesellschaft betroffenen Akteure, wenn die Gesellschaft selbst einen Teil ihres Auftrags an lokale private Milizen weitervergibt, oder wenn sie nicht für einen Staat, sondern für einen multinationalen Konzern agiert. In Verträgen mit privaten Militärfirmen fehlt allzu häufig eine Klausel über die Achtung der Menschenrechte oder des humanitären Völkerrechts. Die Staaten müssen daher dringend einen angemessenen rechtlichen Rahmen verabschieden. Die Verträge zwischen den verschiedenen Parteien sowie die Schulung der Mitarbeiter der Privatgesellschaften müssen auch dazu beitragen, die Gefahren von Verletzungen des humanitären Völkerrechtes, unter denen die von bewaffneten Konflikten betroffenen Gemeinschaften zu leiden hätten, auf ein Mindestmass zu reduzieren.

\section{Privatisierung der Wasserversorgung}

Seit mehreren Jahrzehnten werden die öffentlichen Trinkwasserversorgungsund Abwasseraufbereitungsbetriebe, besonders in den Entwicklungsländern, auf Betreiben der internationalen Finanzinstitutionen teilweise privatisiert. So wurde die Verwaltung von Pumpwerken und Kläranlagen, wie auch das Wasserleitungssystem und die Gebührenerhebung bei den Benutzern im Rahmen von Konzessions- oder Pachtverträgen multinationalen Konzernen anvertraut. Mit diesen Massnahmen wollen die Weltbank und andere Finanzinstitutionen die Effizienz und die Rentabilität des Sektors verbessern.

Der Wassermarkt unterscheidet sich durch verschiedene Besonderheiten von anderen Märkten. Die Rentabilität des Wassersektors hängt insbesondere vom Verkaufspreis pro Kubikmeter Wasser ab, der von der Regierung aus politischen Gründen oft willkürlich plafoniert wird. Im Fall einer humanitären Krise hat der Trinkwasserzugang absolute Priorität, um Epidemien zu verhindern und gerade in den Städten die öffentliche Gesundheit zu bewahren. Deshalb ist es wesentlich, dass die privaten Auftragnehmer ihre Dienstleistungen an die Bevölkerung im Kriegsfall aufrechterhalten, selbst wenn es rein technisch nicht mehr möglich ist, die Gebühren bei den Verbrauchern einzuziehen und somit Gewinne zu erwirtschaften. Die Trinkwasserversorgung der Bevölkerung zu unterbrechen, stellt zudem eine gravierende Zuwiderhandlung gegen das humanitäre Völkerrecht dar.

Da diese Gesellschaften vor allem die Rentabilität im Auge haben, sind sie versucht, sich im Fall eines bewaffneten Konflikts aus dem Markt zurückzuziehen, um Verluste zu vermeiden. Einige Unternehmen beschliessen dennoch im Namen der Unternehmensverantwortung und in einer langfristigen Perspektive, ihre Arbeit trotz der Kriegssituation fortzusetzen. Sie suchen dann nach Mitteln und Wegen, um den Schaden zu begrenzen, unter anderem mit dem Einsatz der für die humanitäre Hilfe und den Wiederaufbau verfügbaren Ressourcen.

Die Geberstaaten und die humanitären Organisationen messen der Garantie der Wasserversorgung der Bevölkerung grösste Bedeutung bei. Somit fragt es sich, ob und inwieweit ein multinationaler Konzern unterstützt werden darf, ohne dass die Mittel und das bereitgestellte Material eine rechtswidrige Subvention darstellen, die den Bestimmungen der Welthandelsorganisation zuwiderläuft und sich weder gegenüber den Steuerzahlern noch der Konkurrenz rechtfertigen 
lässt. Eine Alternative besteht darin, die Mittel mit der Auflage vorzuschiessen, dass sie von den Schulden des Staates gegenüber dem konzessionierten Unternehmen abgezogen werden, sobald wieder Verbrauchergebühren erhoben werden können. Auf alle Fälle sollte die Verantwortung der Unternehmen im Fall eines bewaffeten Konflikts in so lebenswichtigen Bereichen wie der Wasserversorgung in den Konzessionsverträgen viel klarer definiert werden, insbesondere wenn die Weltbank oder andere internationale Organisationen den Privatisierungsprozess fördern. Eine zusätzliche Option wäre die Einführung eines internationalen Mechanismus zur finanziellen Deckung der Kriegsrisiken. Die Privatunternehmen müssten dann die Tätigkeiten in zentralen Bereichen wie der Wasserversorgung auch im Fall eines bewaffeten Konflikts aufrechterhalten, jedoch mit der Gewissheit, die finanziellen Verluste durch eine angemessene Versicherung zu beschränken.

Einige Verantwortliche humanitärer Organisationen lehnen es aus Prinzip ab, mit transnationalen Konzernen in einer Partnerschaft zusammenzuarbeiten. Andere fragen sich, inwiefern sich eine solche Opposition gegen private Betreiber rechtfertigt, zumal die humanitäre Gemeinschaft öffentliche Staatsbetriebe in anderen Krisenländern unterstützt, ohne je ein gründliches Audit durchzuführen, auch wenn bestimmte Staatsbetriebe für ihr Transparenzdefizit oder für die Vergeudung der ihnen anvertrauten Mittel notorisch bekannt sind. Diese verschiedenen Standpunkte spiegeln persönliche Empfindsamkeiten und spezifische Positionen der einzelnen Organisationen wider.

\section{Privatisierung des Haftsystems}

In vielen Ländern werden Haftanstalten vom öffentlichen und vom privaten Sektor partnerschaftlich verwaltet. Dabei werden Dienstleistungen für Häftlinge, wie Verpflegung und Gesundheitsversorgung, an private Firmen ausgelagert. Besonders in angelsächsischen Ländern ${ }^{3}$ werden ganze Haftanstalten ${ }^{4}-$ sogar die Aufsicht und Bewachung - von Privatfirmen wahrgenommen. Eine ausführliche Fachliteratur befasst sich mit diesem Thema und mit den Folgen, insbesondere betreffend die Achtung der Menschenrechte. So wurde zum Beispiel festgestellt, dass das Ziel der Gewinnmaximierung der Privatunternehmen manchmal direkt mit den Auflagen und Zielsetzungen des Haftsystems kollidiert - z.B. den Insassen angemessene Haftbedingungen gewährleisten, die Arbeitskraft der Häftlinge nicht ausbeuten oder die soziale Wiedereingliederung der Häftlinge vorbereiten und fördern.

Im Zusammenhang mit bewaffneten Konflikten handelt es sich bei dieser Art der Privatisierung um eine neuere Entwicklung. Das Thema geriet erst vor kurzem im Zusammenhang mit den Missbräuchen im Gefängnis von Abu Ghraib im Irak in die Schlagzeilen. Wenn die Mitarbeiter eines multinationalen Konzerns gegenüber den Inhaftierten, um die sie sich kümmern sollen, das humanitäre Völkerrecht verletzen, stellen sich mehrere Fragen. Wer ist verantwortlich, wer muss bestraft werden? Der Angestellte, die Firma/das Unternehmen, das Aufsichtsministerium oder alle drei? Inwiefern haften der Herkunftsstaat der Privatgesellschaft und der Staat, in dem die Missbräuche begangen wurden?

3 Australien, Grossbritannien, Vereinigte Staaten und Neuseeland sowie Südafrika.

4 Zum Beispiel Group 4 Securicor; Serco, GEO Group Inc. 
Nach welcher Gerichtsbarkeit und nach welchen Verfahren sollen die Verstösse geahndet/bestraft werden? Sieht der zwischen dem Staat und dem Unternehmen abgeschlossene Vertrag einschlägige Bestimmungen vor? Muss das IKRK seine Beobachtungen zu den Besuchen und den Gesprächen mit den Insassen nur den Staaten übermitteln oder auch den Gesellschaften, die an den Verhören der Gefangenen mitwirken? Wer ist der Hauptansprechpartner für die Verbesserung der Haftbedingungen? Die oben aufgeführten juristischen Überlegungen zu den privaten Militärfirmen liefern einige Antworten. Das IKRK beharrt auch in diesem Fall auf der Verantwortung der Staaten, selbst wenn sie bestimmte Vorrechte an Privatgesellschaften abgetreten haben.

\section{Privatisierung der Gesundheitsdienste}

Kostenlose Gesundheitsversorgung ist für die Patienten in den meisten Krisenländern längst nicht (oder nicht mehr) selbstverständlich. Eine Ausnahme bilden die Gesundheitsleistungen, welche die humanitären Organisationen direkt erbringen. Die Gesundheitsversorgung der ärmeren Bevölkerungsschichten ist jedoch nicht nur in humanitären Krisen prekär. In den meisten Entwicklungsländern entsteht heute ein Zweiklassen-Gesundheitssystem: Private Strukturen bieten hochwertige Pflege an, die sich nur die Reichen leisten können; bankrotte öffentliche Strukturen decken immer öfter einen Teil ihrer Kosten durch die Anrechnung der Pflege ab. Unterbezahlte Angestellte bitten die Patienten sehr häufig mit Forderungen nach Gebühren und offiziösen Honoraren gleich mehrfach zur Kasse. Diese gänzlich informelle Privatisierung „,von innen“ erfolgt ohne jegliche Transparenz. In anderen Fällen einigen sich die öffentlichen Einrichtungen, die Gesundheitszentren und ihre Benutzer auf die Einführung eines Systems kollektiver Vorauszahlung durch eine Versicherungsdeckung, wie dies zum Beispiel in einigen Kommunen von Serbien und Montenegro der Fall ist.

In bewaffneten Konflikten spitzen sich die Bedürfnisse der Bevölkerung und die Verletzlichkeit der ärmsten Menschen zu. Zudem sieht das humanitäre Völkerrecht die Pflicht vor, den Opfern eines bewaffneten Konfliktes den Zugang zur Gesundheitsversorgung zu garantieren. Die formelle und informelle Privatisierung der Krankenpflege erschwert jedoch die Arbeit der humanitären Organisationen, welche die bestehenden Gesundheitsstrukturen meistens nicht ersetzen, sondern unterstützen wollen, und gleichzeitig Personen, deren Gesundheit durch den bewaffneten Konflikt direkt gelitten hat, den Zugang zur medizinischen Versorgung gewährleisten. In akuten Krisensituationen werden die Konfliktopfer vertriebene Bevölkerungen oder Kriegsverletzte - bisweilen nicht medizinisch versorgt, weil sich das Gesundheitspersonal in erster Linie um zahlungskräftige Klienten kümmert, die für die Leistungen aufkommen können, und um die ansässige Bevölkerung, die besser in lokale Netzwerke integriert ist.

Humanitäre Organisationen, die sich bemühen, primäre und sekundäre Gesundheitsstrukturen in Krisenzeiten aufrechtzuerhalten, müssen unbedingt verstehen, wie diese Strukturen funktionieren, und Ratschläge zur administrativen und finanziellen Verwaltung erteilen können, um besser in der Lage zu sein, sinnvolle und nachhaltige Unterstützung zu leisten. Die Erfahrung hat gezeigt, dass humanitäre Helfer zwar zumeist in akuten Notsituationen einschreiten, dass ihre Unterstützung der lokalen Gesundheitssysteme aber dazu neigt, sich 
über viele Jahre hinzuziehen. Eine dauerhafte Intervention erfordert immer umfassendere Kenntnisse in Anbetracht der Besonderheiten des „Gesundheitsmarktes" und der Versicherungssysteme (z.B. Teilprivatisierung, mit oder ohne Versicherungsmechanismus, asymmetrische Information, moralisches Risiko, negative Auslese, usw.).

\section{Schlussfolgerungen}

Die humanitären Krisen rücken - aufgrund ihrer besonderen Merkmale und der dramatischen Konsequenzen für die betroffenen Gemeinschaften - die wesentlichen Fragen in den Vordergrund, die durch die Privatisierung und die Auslagerung der öffentlichen Basisdienste aufgeworfen werden.

Die erste Frage betrifft die Vereinbarkeit zwischen dem Ziel der Gewinnmaximierung - die Priorität der Privatunternehmen - und der Erhaltung der Sicherheit und der öffentlichen Gesundheit - ein Anliegen der Staaten. Auf dem Markt als Institution kommen Angebot und Nachfrage sowie der Wettbewerb frei zum Tragen. Das Ziel ist eine optimale Ressourcenallokation sowie die Förderung von Kreativität und Innovation, um die Nachfrage der Verbraucher zu decken bzw. neue Bedürfnisse zu wecken. Selbstverständlich kennt der Markt an sich keine Ethik: die Mechanismen von Angebot und Nachfrage greifen sowohl auf dem Aktienmarkt und dem Kaffeemarkt, als auch auf dem Markt der Kinderprostitution oder dem Kokainmarkt. Es ist Aufgabe des Staates, bestimmte Märkte aus Gründen der öffentlichen Ordnung, Sicherheit, Moral oder Gesundheit u.a. in einen Rahmen bzw. unter ein Verbot zu stellen. Der Staat hat auch die Pflicht, dem Druck von Privatfirmen zu widerstehen, falls sie die Behörden dazu drängen sollten, die Nachfrage nach ihren Dienstleistungen anzukurbeln sprich im Fall der privaten Militärfirmen einen bewaffneten Konflikt anzuzetteln, fortzusetzen oder zu verschärfen.

Ausserdem ist es wesentlich, dass die Verträge zur Weitergabe von Aufträgen zwischen den Regierungen und Privatfirmen in so sensiblen Bereichen wie der Wasserversorgung oder der Gefängnisverwaltung die Verantwortungen und Pflichten der Parteien im Fall höherer Gewalt (z.B. bewaffneter Konflikt) in präzisen Klauseln regeln. Die Verträge sollten auch wirtschaftliche und finanzielle Anreizmechanismen vorsehen, um die vorrangige Berücksichtigung der humanitären Anliegen zu garantieren. Andernfalls hätten die privaten Gesellschaften ein leichtes Spiel, sich auf die Verträge mit den Behörden zu beziehen, die dann keinerlei diesbezügliche Bestimmung enthielten.

Die zweite Frage betrifft die Achtung des Völkerrechts durch Privatunternehmen und die Verantwortung des Staates, wenn durch die Tätigkeiten dieser Unternehmen die Achtung des humanitären Völkerrechts gefährdet ist. Die Staaten haften, wie erwähnt, für die Handlungen aller Personen und Instanzen, die zur Ausübung von Rechten der öffentlichen Gewalt ermächtigt sind oder die (de facto) auf Weisung oder unter Kontrolle des Staates handeln. Jedoch ist das Risiko, dass die Staaten sich durch die Hintertür der Privatisierung und der Auslagerung aus ihrer völkerrechtlichen Verantwortung stehlen, durchaus real und sollte aufmerksamer überwacht werden.

Die dritte Frage betrifft die ökonomische Rationalität dieser Entwicklung, das Hauptargument der verantwortlichen Politiker, die den Privatisierungen wegen 
der davon erhofften Einsparungen das Wort reden. Die Bilanz der Privatisierung öffentlicher Dienstleistungen bleibt jedoch umstritten. Eine von Abt Associates, - einer nordamerikanischen Consultingfirma, die Beratungsdienste im Bereich Wirtschaftsreformen und Privatisierung anbietet, und der in dieser Hinsicht bestimmt keine negativen Vorurteile nachgesagt werden können, - 1998 veröffentlichte Studie ${ }^{5}$ kommt zum Schluss, dass die verfügbaren Analysen und Daten nicht beweisen, dass der Einsatz von Privatfirmen im Gefängniswesen zu Einsparungen führt. Die Organisation „War on Want" proklamiert noch kategorischer, dass die Privatisierung zahlreicher öffentlicher Dienstleistungen die Armut in den Entwicklungsländern verschärft habe, und dass die britische Entwicklungshilfeagentur mit der Unterstützung dieser Reformen nicht die Armut in den Empfängerländern gelindert, sondern Konsulenten und britische Privatfirmen bereichert habe ${ }^{6}$. Die Argumente zu den ökonomischen Vorteilen verschiedener institutioneller Übereinkommen zwischen dem öffentlichen und dem privaten Sektor müssen folglich genau untersucht werden. Dabei sind politische, soziale und weitere Überlegungen mit zu berücksichtigen.

Die humanitären Organisationen sollten sich bemühen, den Beitrag und die Arbeitsweise der privaten Dienstleistungsanbieter besser zu verstehen. Das würde ihre Fähigkeiten verbessern, mit diesen neuen Akteuren in humanitären Krisenfällen zu verhandeln - zum Wohl der betroffenen Gemeinschaften. Der Austausch zwischen humanitären Organisationen und Privatunternehmen darf jedoch in keinem Fall die Staaten aus ihren völkerrechtlichen Verpflichtungen entlassen, besonders nicht in „Privatisierungssektoren“ wie Ausbildung von Streitkräften, Gefängnisverwaltung, Trinkwasserversorgung und medizinische Versorgung.

5 „Private Prisons in the United States: An Assessment of Current Practice“, Cambridge (Massachusetts), Abt Associates Inc., 16.7.1998, S. 56, zitiert in The Sentencing Project. „Prison Privatization and the Use of Incarceration“, Washington, D.C. ; Überarbeitung vom September 2004, <http://www. sentencingproject.org/pdfs/1053.pdf>.

6 Duncan Campbell, «UK accused of using aid to promote privatisation», The Guardian, 27.9.2004. 